\title{
Lessons from Participatory Design with Adolescents on the Autism Spectrum
}

Miriam Madsen

MIT Media Lab

20 Ames St.

Cambridge, MA 02139

mish@mit.edu

Rana el Kaliouby

MIT Media Lab

20 Ames St.

Cambridge, MA 02139

kaliouby@media.mit.edu

Micah Eckhardt

MIT Media Lab

20 Ames St.

Cambridge, MA 02139

micahrye@mit.edu

Mohammed E. Hoque

MIT Media Lab

20 Ames St.

Cambridge, MA 02139

mehoque@mit.edu

Copyright is held by the author/owner(s).

CHI 2009, April 4-9, 2009, Boston, MA, USA.

ACM 978-1-60558-247-4/09/04.
Matthew S. Goodwin

MIT Media Lab

20 Ames St.

Cambridge, MA 02139

mgoodwin@media.mit.edu

Rosalind Picard

MIT Media Lab

20 Ames St.

Cambridge, MA 02139

picard@media.mit.edu

\begin{abstract}
Participatory user interface design with adolescent users on the autism spectrum presents a number of unique challenges and opportunities. Through our work developing a system to help autistic adolescents learn to recognize facial expressions, we have learned valuable lessons about software and hardware design issues for this population. These lessons may also be helpful in assimilating iterative user input to customize technology for other populations with special needs.
\end{abstract}

\section{Keywords}

Autism, ASD, adolescents, design, user interface, emotion detection, facial expression analysis

\section{ACM Classification Keywords}

H5.2. User Interfaces: User-Centered Design.

\section{Introduction}

Autism Spectrum Disorder is a collection of developmental disabilities characterized by qualitative impairments in socialization, communication, and circumscribed interests, including stereotypical behavior patterns and rigidity to changes in routine [1]. In addition to these core characteristics, individuals on the 
autism spectrum often present with intellectual [2], sensory [3], and motor impairments [4]. Although all of these symptoms are common, autism is highly heterogeneous and idiosyncratic; each person on the autism spectrum has his or her unique profile of difficulties and strengths.

As described in greater detail in the current paper, the following three issues are important to address when designing technologies for this population: (1) cognitive impairments, including memory problems and belowaverage reading ability; (2) sensory challenges related to atypical sensory processing and perceptual experiences; and (3) motor issues, including gross and/or fine motor control difficulties.

We chose to implement an iterative, participatory design process to address these particular difficulties as we create assistive technologies for this population. The following sections explore design challenges we have encountered and details ways we have overcome them.

\section{Goals and Contributions of the Research}

In this work, we focus on the social difficulties prominent in autism by providing a forum in which individuals can improve their interactions. We have developed iSET (Interactive Socio-Emotional Toolkit), a combination of hardware and software that assists a user's recognition, understanding, and expression of self and others facial affect and emotions [5].

iSET works in two ways. Using state-of-the-art facial expression inference algorithms [6], iSET allows realtime analysis, recording, and tagging of facial

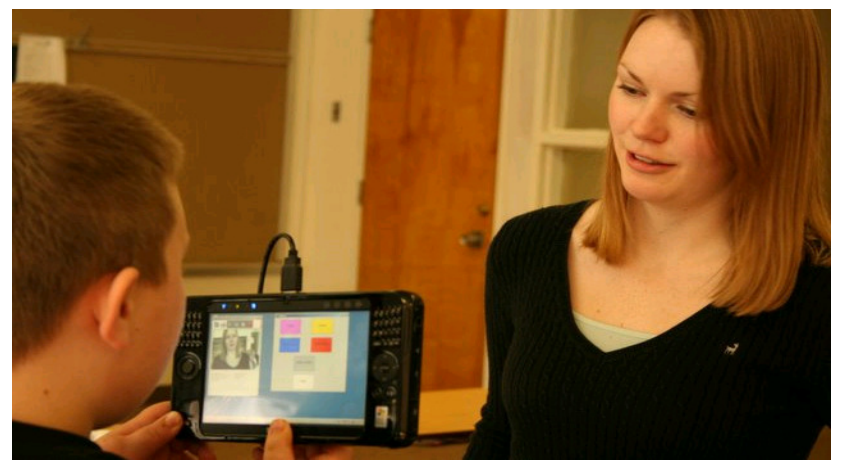

figure 1: A student on the autism spectrum using iSET with his teacher to capture, tag, and analyze facial expressions.

expressions. As shown in Figure 1, the system detects, recognizes, and visualizes a range of facial expressions.

iSET also has an offline component where a student and teacher can record naturally-evoked facial expressions and analyze them later. In the setting described in this paper, iSET was primarily used in an unstructured way, although we asked participants to try to elicit and record certain facial expressions representing specific emotions from their teachers and classmates.

Our goals are twofold: (1) develop in-situ technologies for individuals on the autism spectrum that are fun, comfortable to use, and capable of improving social interactions; and (2) demonstrate how insights from adolescents with a disability and their caregivers can greatly improve the technology with each iteration of development through a series of participatory design sessions. 


\section{Iterative Participatory Design Sessions} The participatory design sessions took place at [anonymized], a non-profit school that provides evaluative, therapeutic, and educational programs for individuals with autism and other developmental disorders. Four sessions were carried out at [anonymized] over the course of four months with seven verbal adolescents on the autism spectrum and their teachers. The participants were all males aged 1017 years.

The goal of these sessions was to identify usability issues that our participants had with iSET and make iterative changes to the hardware and software that accommodate for these issues. The sessions were challenging since participants were not always able to articulate the difficulties they had with the system. Thus, we used a combination of methods to document usability issues. First, we had two clinical assistants familiar with the students observe what each participant found engaging, as well as note specific difficulties. Second, the sessions were videotaped to allow retrospective review. Finally, we interviewed our participants and their teachers after the sessions.

\section{Results}

The major design lessons we learned during the participatory sessions can be divided into three areas:

(1) adaptation of the hardware's form factor; (2) modification to the graphical user interface; and (3) enhancement of the user experience. For each of these areas we review feedback compiled over the course of the sessions and describe the iterative design changes we made to incorporate our users' feedback.

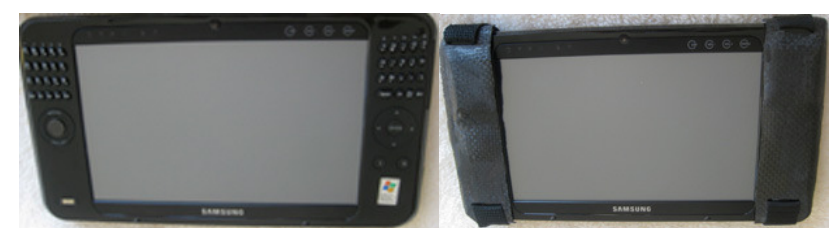

figure 2: The Ultramobile's keyboard (left) and our fabricated external shields (right).

\section{Form Factor Adaptation}

The first iteration of the device-an ultra-mobile Samsung computer-provided access to a keyboard and trackpoint to navigate iSET. During the sessions we found that the keyboard was distracting for several participants (i.e., they became preoccupied with pressing the keys on the keyboard), and that the device was difficult to hold steadily. To overcome these issues we fabricated external shields to hide the controls and used the device's touch screen for user input (see figure 2). The external shield's attachment straps also seemed to make the device easier to hold.

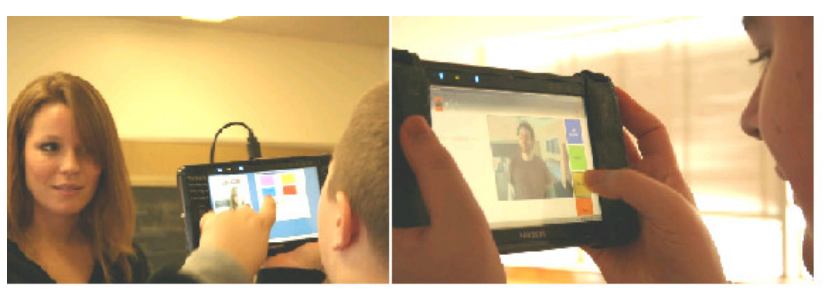

figure 3: A re-design of the interface (shown on the right) allowed participants to interact with iSET using their thumbs.

To facilitate easy navigation, we adjusted the placement and size of the touch screen buttons to allow participants to use their thumbs (figure 3 ). We also identified the need for a neck strap that would further 
enable users to hold the device steadily and prevent it from being dropped.

\section{Graphical User Interface Improvement}

Many improvements were made to the graphical user interface over the course of the study to facilitate a more intuitive and engaging experience with the technology. As shown in figure 4 , the initial version of iSET showed live video, 22 facial feature points if a face was detected, and color-coded bubbles that indicate that a particular expression was detected.

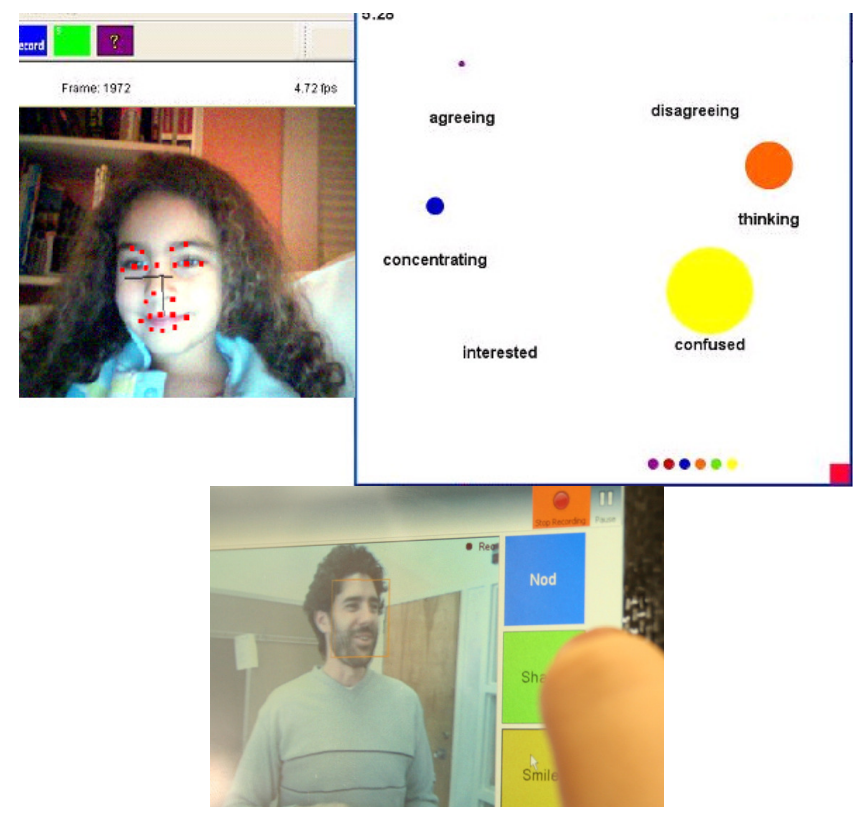

figure 4: The initial version of iSET highlighted 22 facial feature points. Later, this highlighting mode changed to a bounding box, which obscured the face less while still clearly indicating that the system was processing the participant's face.
We quickly found that highlighting the feature points was confusing for our users, and often occluded the facial expressions we were trying to teach them. We therefore replaced the feature points with a simple bounding box around the face.

Based on iterative feedback, interactive affective tagging components were added and the interface was made customizable to suit each participant's interests and difficulties in recognizing particular facial expressions. For example, some participants were good at recognizing happiness, sadness, and anger. For those participants, we were able to instantly customize the interface to handle a more challenging set of affect labels, such as confusion and excitement. Several of our participants struggled with reading the words on the tagging buttons. Therefore, we replaced the text with representative images to accommodate for reading difficulties - a standard strategy for teaching autistic persons to interpret and express emotions. Our system reinforced current strategies by using the same BoardMaker icons in use by the students' teachers for teaching emotions.

As shown in figure 5, the final version allows for custom control of the tagging components, where words, pictures, and colors on the tagging buttons can be customized for the individual.

User Experience Improvement

At the start of each session participants were presented with iSET and given an explanation of what it was and how it could be used. Participants were then encouraged to use iSET in an unstructured way. This allowed us to observe differences between our expectation of how the device should be used and how 
participants actually used it. Several new interaction scenarios were generated from these observations.
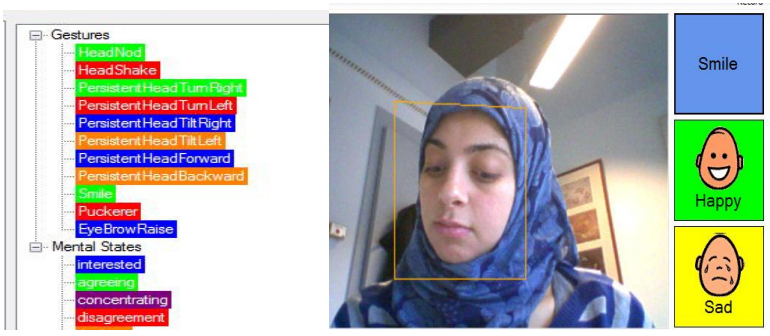

figure 5: Fully customizable tagging components of iSET. Left: choice of facial expressions and colors are customizable through menu options. Right: tagging buttons including choice of text and image can be customized by the participant.

For instance, we found that some participants enjoyed playing an "expression hunt" game where they were asked to identify people exhibiting particular

expressions. Other participants who typically socialize very little began a spontaneous "movie actor and director" game that increased interactions in very expressive ways. During this game, two participants took turns playing actor and director; the actor would make the expressions and the director's job was to capture them. Over several sessions, a participant expressed his desire to recreate his favorite movie,

"Watership Down". These innovations were recorded and encouraged by our researchers, who helped the participants think of new ways to use the system.

\section{Discussion}

While our technology is still under development, it appears to be beneficial in helping autistic individuals interact with others while learning about affective information. Numerous accommodations were made to enhance the form factor, user interface, and overal user experience. Below we revisit accommodations made for this population's cognitive impairments, sensory challenges, and motor issues while using our system.

\section{Accommodating Cognitive Impairments}

Multiple revisions to the software were made to make it easier for participants with reading difficulties to use

(see figure 6). These refinements included placement of text, size of text, dynamic labeling of buttons, and graphically-labeled buttons. Memory/attention deficits were addressed by encouraging students to have more active interactions, and to choose their own activities, both of which appeared to increase participants' interest in iSET.

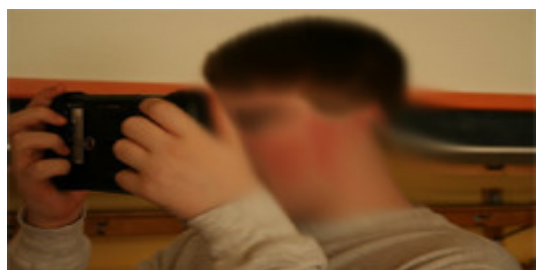

figure 6: Text size adaptations made the system easily readable for users with vision difficulties.

\section{Accommodating Sensory Challenges}

The main sensory challenges encountered during this study were tactile and auditory distraction caused by the keyboard and mouse controls on the ultramobile Samsung Q1. The participants often became fixated with pressing the controls. This both distracted them from the intended use of the device and resulted in either accidently closing the iSET application or turning 
the computer off. This problem was solved by adding custom control shields.

\section{Accommodating Motor Issues}

To address problems with gross motor abilities, the control shields and accompanying straps made the device easier to hold. Making the on-screen buttons larger accommodated for problems with fine motor skills. The buttons were also placed to the right-most side of the screen, allowing participants to use their thumbs to interact with the system.

\section{Conclusion}

The participatory design sessions provided insights into the usability of our system and were critical to the development of our technology, underscoring the importance of including people on the autism spectrum and their caregivers in the design process of new technologies. For these technologies to be effective, they need to accommodate the cognitive, sensory, and motor difficulties of their users. Since persons on the autism spectrum frequently present with a range of cognitive and physical impairments, many of the lessons learned in this work could be applied to other populations with similar disabilities. The use of participatory design is an important part of developing technologies that address the specific needs of underrepresented groups, such as those on the autism spectrum, and others with cognitive, sensory perceptual, and motor impairments.

\section{Acknowledgements}

This material is based upon work supported by the National Science Foundation under Grant No. 0555411 and the Things That Think Consortium. Any opinions, findings, and conclusions or recommendations expressed in this material are those of the author(s) and do not necessarily reflect the views of the National Science Foundation. The authors would like to especially thank the participants and staff at the Groden Center for participating in our pilot, as well as Youssef Kashef and Abdelrahman Mahmoud for help with updating the system between sessions. The hardware was provided by Samsung, and the tracker for the face analysis software was provided by Google.

\section{References}

[1] American Psychiatric Association. (2000). Pervasive developmental disorders. Diagnostic and statistical manual of mental disorders, DSM-VI-TR, (pp. 69-70). Washington, D.C.

[2] Prior, M. \& Ozonoff, S. (1998). Psychological factors in autism. In F. R. Volkmar (Ed.) Autism and Pervasive Developmental Disorders (pp. 64-108). Cambridge, England: Cambridge University Press.

[3] Iarocci, G. and J. McDonald, Sensory Integration and the Perceptual Experience of Persons with Autism. Journal of Autism and Developmental Disorders, 2006. 36(1): p. 77-90.

[4] Piek, J. and M. Dyck, Sensory-motor deficits in children with developmental coordination disorder, attention deficit hyperactivity disorder and autistic disorder. Human Movement Science, 2004. 23(3-4): p. 475-488.

[5] Madsen, M., et al., Technology for just-in-time in-situ learning of facial affect for persons diagnosed with an autism spectrum disorder, in Proceedings of the 10th international ACM SIGACCESS conference on Computers and accessibility. 2008, ACM: Halifax, Nova Scotia Canada. p. 19-26.

[6] el Kaliouby, R. and P. Robinson, Real-time Inference of Complex Mental States from Facial Expressions and Head Gestures, in Real-Time Vision for Human-Computer Interaction. 2005, Springer-Verlag. p. 181-200. 